\title{
A quasi-periodic route to chaos in a parametrically driven nonlinear medium
}

\author{
Ana M. Cabanass ${ }^{\mathrm{a}, *}$, Ronald Rivas ${ }^{\mathrm{b}, \mathrm{c}}$, Laura M. Pérez ${ }^{\mathrm{b}}$, Javier A. Vélez ${ }^{\mathrm{b}, \mathrm{d}}$, Pablo Díaz ${ }^{\mathrm{e}}$, \\ Marcel G. Clerc ${ }^{\mathrm{f}}$, Harald Pleiner ${ }^{\mathrm{g}}$, David Laroze ${ }^{\mathrm{b}, * *}$, Boris A. Malomed ${ }^{\mathrm{h}}$ \\ ${ }^{a}$ Sede Esmeralda, Universidad de Tarapacá, Av. Luis Emilio Recabarren 2477, Iquique, Chile \\ ${ }^{b}$ Instituto de Alta Investigación, Universidad de Tarapacá, Casilla 7D, Arica, Chile \\ ${ }^{c}$ Departamento de Física, Universidad Nacional Experimental Francisco de Miranda, Coro, Venezuela \\ ${ }^{d}$ Centro de Nanociencia y Nanotecnología, CEDENNA, Av. Libertador Bernardo O'Higgins 3363, \\ Santiago, Chile \\ ${ }^{e}$ Departamento de Ciencias Físicas, Universidad de La Frontera, Casilla 54-D, Temuco, Chile \\ ${ }^{f}$ Departamento de Física and Millennium Institute for Research in Optics, Facultad de Ciencias Físicas y \\ Matemáticas, Universidad de Chile, Casilla 4873, Santiago, Chile \\ ${ }^{g}$ Max Planck Institute for Polymer Research, D 55021 Mainz, Germany \\ ${ }^{h}$ Department of Physical Electronics, School of Electrical Engineering, Faculty of Engineering, Tel Aviv \\ University, Tel Aviv 69978, Israel
}

\begin{abstract}
Small-sized systems exhibit a finite number of routes to chaos. However, in extended systems, not all routes to complex spatiotemporal behavior have been fully explored. Starting from the sine-Gordon model of parametrically driven chain of damped nonlinear oscillators, we investigate a route to spatiotemporal chaos emerging from standing waves. The route from the stationary to the chaotic state proceeds through quasiperiodic dynamics. The standing wave undergoes the onset of oscillatory instability, which subsequently exhibits a different critical frequency, from which the complexity originates. A suitable amplitude equation, valid close to the parametric resonance, makes it possible to produce universe results. The respective phase-space structure and bifurcation diagrams are produced in a numerical form. We characterize the relevant dynamical regimes by means of the largest Lyapunov exponent, the power spectrum, and the evolution of the total intensity of the wave field.
\end{abstract}

\section{Introduction}

A natural way to pump energy into various dynamical systems is offered by the use of direct or parametric resonances [1]. Direct resonances were exploited at the dawn of modern physics by Galileo [2] in the characterization of a pendulum. Parametric resonances were highlighted by the pioneering works of Faraday [3] in the study of

\footnotetext{
${ }^{*}$ Corresponding author

${ }^{* *}$ Corresponding author

Email addresses: ana.cabanas.plana@gmail.com (Ana M. Cabanas), dlarozen@uta.cl (David Laroze)
} 
vibration modes of a water tank. Theoretical and experimental studies of parametrically forced nonlinear systems are relevant to many settings in physics, chemistry, biology, and engineering. Due to the competition between injection and dissipation of energy, the driven systems demonstrate complex behavior, leading to the formation of a wide range of spatiotemporal structures, such as localized ones, extended patterns, and chaotic states, among others [4-11]. One of the most generic scenarios of the selforganizing behavior is pattern formation. With the increase of the energy-injection rate, basic stationary patterns may become unstable and bifurcate into a great variety of more complex ones $[6,7,11-22]$. In particular, a manifestation of the complex spatiotemporal dynamics is time-aperiodic behavior, as observed, inter alia, in fluids [23-27], chemical waves [28], cardiac fibrillation [29], granular matter [30], electroconvection [31], and photonic devices [32-34]. In the broad variety of physical setups exhibiting complex spatiotemporal behaviors, routes leading to the emergence of spatiotemporal chaos are not fully understood yet, as analytical investigation of the underlying nonlinear partial differential equations is a challenging problem. Diverse routes have been identified for the transition from order to disorder in wave settings, such as defectmediated turbulence [35-37], onset of spatiotemporal chaos in Galilean-invariant systems [38], spatiotemporal intermittence [39], quasi-periodicity of travelling [40] and standing waves [14], crisis of spatiotemporally chaotic saddles [41-43], spatiotemporal chaos which sets in via stationary branching shocks and holes [44], and the phase turbulence [45].

A generic model that describes periodically forced systems is based on the parametrically driven damped nonlinear Schrödinger (PDDNLS) equation [46-48]. This amplitude equation produces a variety of temporal behaviors, including stationary, periodic, and chaotic regimes, such as Faraday waves [3, 49-51], single-soliton [47, 5255] and two-soliton [56, 57] states, and spatiotemporal chaos [47, 58]. Remarkable hydrodynamic modes are excited by the parametric instability in the form of standing (Faraday) waves on the surface of a vertically vibrated Newtonian fluid [3]. These standing waves respond strongest at a half of the forcing frequency (the $2: 1$ resonance) [59]. The basic PDDNLS equation describes parametric instabilities close to the $2: 1$ resonance in weakly dissipative systems [61]. However, this equation in its simplest form is not able to produce certain phenomena, such as stable localized modes that connect a uniform oscillatory state to an extended wave [60], or domain walls between uniform oscillations with opposite phases [61-64]. To study the existence and properties of a broad class of dynamical states, a generalized PDDNLS equation, produced by a systematic expansion procedure, can be used. It adequately accounts for diverse types of the dynamical behavior, such as fronts and confined patterns [65].

This work aims to investigate dynamical scenarios based on standing waves in a parametrically driven chain of pendula, which is considered in the continuum approximation. Starting from the respective parametrically-driven damped sine-Gordon model, it is shown that spatiotemporal chaos can emerge from standing waves. The chaos appears as an extension of quasi-periodic dynamics. Namely, changing parameters of the model, we observe that the standing wave exhibits an oscillatory instability. Further variation of the parameters leads to a secondary oscillatory instability, which eventually gives rise to the spatiotemporal chaos. The generalized PDDNLS equation, valid close to the parametric resonance, allows to systematically investigate the emer- 
gence of the complex behavior in a numerical form. The largest Lyapunov exponent, power spectrum, and time evolution of the total norm of the wave field are computed to characterize the transitions between stationary, quasi-periodic, and chaotic dynamical states.

The manuscript is organized as follows. The physical model, the nonlinear analysis and the dynamical indicators used to describe the system are presented in Sec. 2. Results produced by systematic numerical investigation of the patterns and characterization of the dynamical behavior of the system are reported in Sec. 3. Finally, conclusions are formulated in Sec. 4.

\section{The parametrically driven damped sine-Gordon equation and the nonlinear- Schrödinger approximation}

We start by consideration of a chain of parametrically driven damped pendula (an extended Scott's model [49]), described in the continuum limit by the parametrically driven damped sine-Gordon equation [50]:

$$
\ddot{\theta}=-\left[\omega_{0}^{2}+\gamma_{0} \sin (\omega t)\right] \sin \theta-\mu_{0} \dot{\theta}+\kappa \partial_{x x} \theta,
$$

where $\theta(x, t)$ is the angle between the pendulum at position $x$ and the vertical axes at time $t$, the overdot stands for the time derivative, $\omega_{0}$ is the eigenfrequency of the pendulum, $\gamma_{0}$ and $\omega$ are the amplitude and frequency of the parametric forcing, $\mu_{0}$ is the damping coefficient and $\kappa$ the coefficient of the elastic coupling. In the following, by means of rescaling of $x$ and $t$ we fix $K=\omega_{0}=1$. Figure 1 illustrates different types of dynamical oscillatory states in the system.

The trivial solution $\theta(x, t)=0$ can be made unstable by the parametric forcing with the frequency close to the resonant (double) value,

$$
\omega=2\left(\omega_{0}+v\right)
$$

where $v$ is a detuning parameter, with $|v| \ll \omega_{0}$. The commonly known analysis [1] produces the stability boundary (the so-called Arnold tongue), in the form of

$$
v^{2}+\left(\mu_{0} / 2\right)^{2}=\left(\gamma_{0} / 4\right)^{2},
$$

depicted in Fig. 2 by the dashed black hyperbola in the parameter plane of the detuning and forcing strength. While crossing the boundary, the destabilization occurs through a supercritical (subcritical) bifurcation at positive (negative) values of the detuning [61], uniform oscillations taking place inside of the "tongue" [64]. The corresponding power spectrum, displayed in panel d) of Fig. 1 shows well-defined peaks determined by the forcing. The respective inset shows the phase portrait corresponding to a closed limit cycle.

In the Arnold tongue, the uniform oscillations are observed at small detuning, as illustrated in Fig. 2 by green circles. Making the detuning more negative, the uniform oscillations develop a supercritical oscillatory spatial instability with a nonzero wavenumber [6]. It is characterized by emergence of a novel frequency, which is not commensurate with the frequency of the uniform oscillations. The instability gives rise 

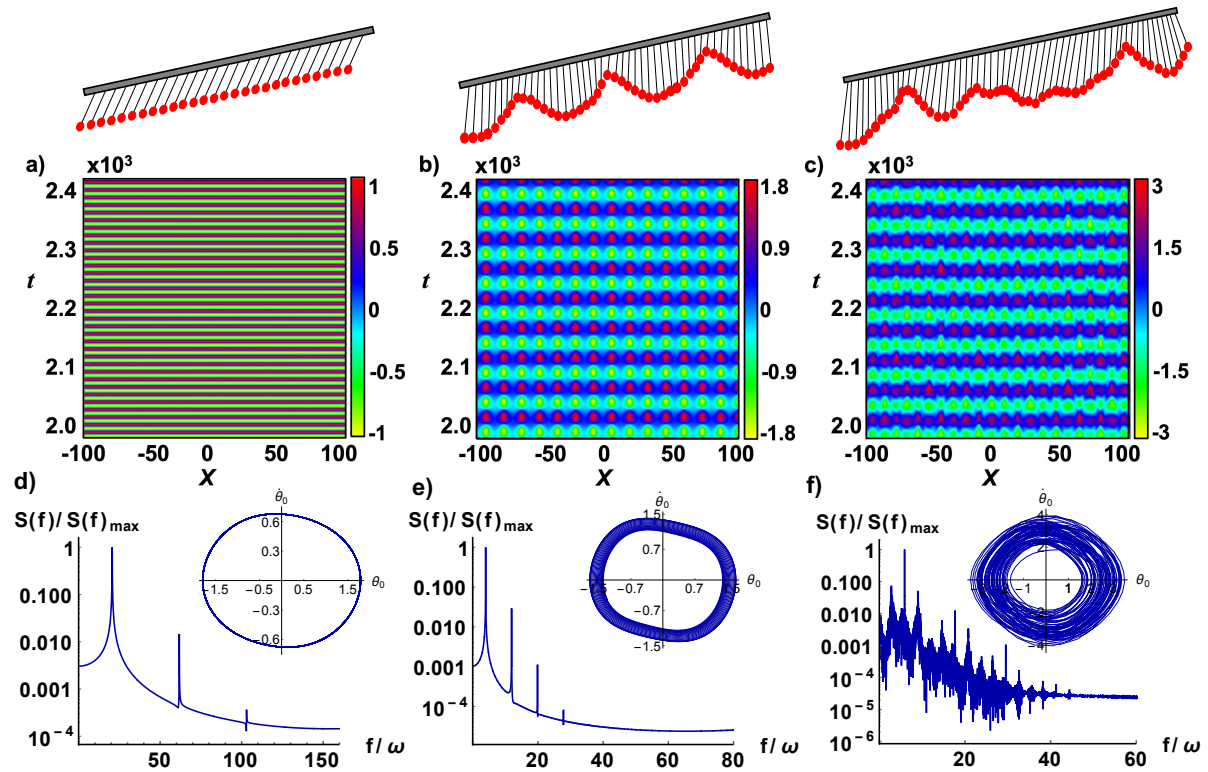

Figure 1: Spatiotemporal dynamical behavior produced by the parametrically driven damped sine-Gordon equation (1). Color-coded spatiotemporal distributions of the continuous field, $\theta(x, t)$, are displayed, with time interval $\Delta \tau=2000$, in the middle row, parallel to typical configurations of the chain of coupled pendula shown in the top row, which is approximated by Eq. (1) in the continuum limit. a) A spatially uniform state obtained at the amplitude and detuning of the parameteric drive $\gamma_{0}=0.85$ and $v=-0.02$ (see Eq. (2)), with the largest Lyapunov exponent $\lambda_{\max }=-0.037$. b) A regular spatially periodic pattern at $\gamma_{0}=0.40$ and $v=-0.05$, with $\lambda_{\max }=-0.041 \mathrm{c}$ ) A chaotic state at $\gamma_{0}=0.61$ and $v=-0.08$, with $\lambda_{\max }=0.055$. Bottom panels d), e), and f) show normalized Fourier spectra $S(f)$ and phase portraits in the plane of $(\theta, \dot{\theta})$ at a fixed spatial point $(x=0)$, plotted for a), b) and c), respectively. Other parameters in Eq. (1) are $\omega_{0}=1, \mu_{0}=0.1$, and $\kappa=1$.

to standing waves, as shown in panels b) and e) of Fig. 1. Note that in the respective phase portrait the limit cycle transforms into a torus. These standing waves are denoted by red triangles in the diagram of states displayed in Fig. 2. Further decreasing the detuning, the standing wave develops a complex spatiotemporal behavior, as illustrated in panels c) and e) of Fig. 1. It is seen that the temporal spectrum at a fixed spatial point now represents dynamical chaos, and, likewise, the phase portrait corresponds to a strange attractor [8]. Such a complex spatiotemporal behavior, denoted by blue squares in Fig. 2, is observed in a broad region of the Arnold tongue.

To characterize the dynamical nature of the complex waves, we use the largest Lyapunov exponent $\lambda_{\max }$ [66]. First, $\lambda_{\max }<0$ corresponds to a stable equilibrium state, a uniform or patterned one. On the contrary, $\lambda_{\max }>0$ implies chaotic dynamics, with exponential sensibility to initial conditions. The case of $\lambda_{\max }=0$ corresponds to quasi-periodic behavior or complex behavior with non-exponential sensitivity. Figure 3 shows $\lambda_{\max }$ as a function of detuning $v$ (see Eq. (2)) for different values of the drive's strength, $\gamma_{0}=\{0.4,0.5,0.6,0.7\}$. The plots make it possible to distinguish regular and chaotic behavior, which, as said above, correspond to $\lambda_{\max }<0$ and $\lambda_{\max }>0$, 


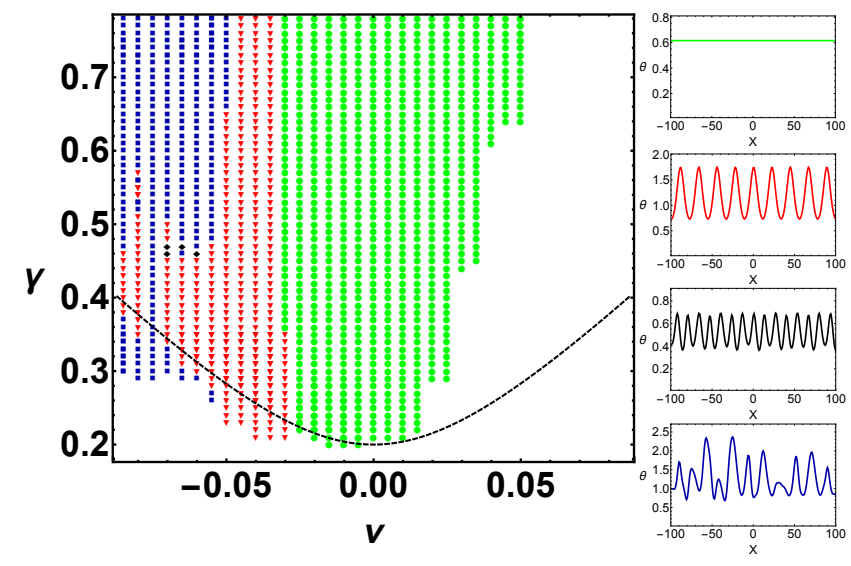

Figure 2: The diagram of dynamical states in the $(\gamma, v)$ parameter plane of Eqs. (1) and (2). The dashed curve represents the instability boundary (Arnold tongue) given by Eq. (3). Green circles $(\bullet)$ represent spatially uniform states, red triangles ( $\mathbf{\nabla})$ denote standing waves, blue squares ( $\mathbf{\square})$ chaotic waves, and black diamonds ( $\bullet$ ) correspond to quasi-periodic states with small amplitudes. Here and in Fig. 5 below, the trivial state, $\theta \equiv 0$, occurs in the white area. Right panels display snapshots of the respective spatial patterns. The fixed parameters are the same as in Fig. 1.

respectively.

Thus, the parametrically driven damped sine-Gordon equation (1) provides the transition from the spatially uniform oscillations to the spatiotemporal chaos, via the intermediate spatially periodic pattern. To achieve a better grasp of these dynamical regimes, in the next section we develop analysis under conditions of weak nonlinearity and slowly-varying amplitude, close to the parametric resonance.

\subsection{The weak-nonlinearity analysis}

The above model, based in the sine-Gordon equation, can be essentially simplified for small-amplitude solutions, whose main harmonic frequency is close to the eigenfrequency, $\omega_{0}$. To this end, the following ansatz is adopted [61]:

$$
\theta(x, t)=\psi(\tau, X) e^{-i \omega \tau / 2}+\text { c.c. }+ \text { h.o.t., }
$$

where $\psi(\tau, X)$ is a slowly complex envelope amplitude, $\tau \equiv t / 4, X \equiv x / \sqrt{2}$, while c.c. and h.o.t. stand for the complex conjugate and higher-order terms, respectively. Close to the $2: 1$ parametric resonance, the frequency is $\omega=2(1+v)$, as per Eq. (2). The substitution of ansatz (4) in (1) yields, in the first approximation, the PDDNLS equation [47], which is the basic model of the driven systems close to the $2: 1$ parametric resonance:

$$
\frac{\partial \psi}{\partial \tau}=-i \nu \psi-i|\psi|^{2} \psi-i \frac{\partial^{2} \psi}{\partial X^{2}}-\mu \psi+\gamma \bar{\psi},
$$

where $\mu \equiv \mu_{0} / 2, \gamma \equiv \gamma_{0} / 4$, and $\bar{\psi}$ is the complex conjugate of $\psi$. This equation is known to produce stationary, time-periodic, or chaotic solutions, including Faraday 
waves [50, 62], soliton-like modes [47, 52], two-soliton [56, 57] and soliton-antisoliton [67] bound states, and spatiotemporal chaos [47, 58].

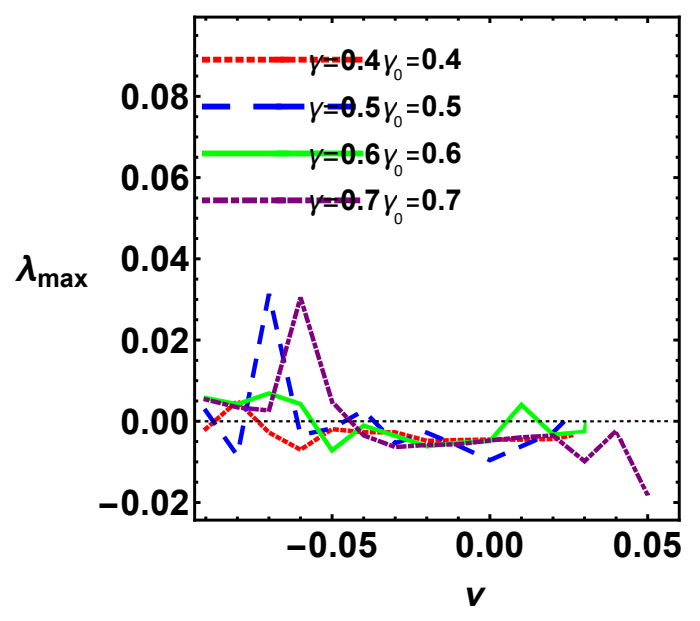

Figure 3: The largest Lyapunov exponent $\lambda_{\max }$ vs. detuning $v$ (see Eq. (2)), at different values of the strength of the parametric forcing: $\gamma_{0}=(0.4,0.5,0.6,0.7)$. The other parameters are the same as in Fig. 2.

The PDDNLS equation admits different spatially uniform solutions. The instability boundary of the trivial one, $\psi=0$, is

$$
\gamma^{2}=\mu^{2}+v^{2}
$$

cf. Eq. (3). In addition, the trivial solution suffers an instability which sets in at $\gamma=\mu$ for the positive detuning $[50,68]$. Nontrivial uniform solutions of Eq. (5) are

$$
\psi_{ \pm, \pm}= \pm x_{0}\left(1 \pm i y_{0}\right)
$$

where $x_{0}=\sqrt{(\gamma-\mu)(\phi-v) / 2 \gamma}$ and $y_{0}=\sqrt{(\mu-\gamma) /(\mu+\gamma)}$ with $\phi \equiv \sqrt{\gamma^{2}-\mu^{2}}$ and two independent signs \pm . Note that the uniform solutions emerge at $\gamma=\mu$ through a saddle-node bifurcation. They are unstable in the parameter space of Eq. (5), with the exception of the case of zero detuning $(v=0)$, in which case $\psi_{ \pm,+}$is marginally stable [63].

In fact, the underlying sine-Gordon equation (1) may have stable solutions for uniform oscillations. To provide this possibility, as well as the existence of more complex states that bifurcate from the uniform oscillations, it is necessary to proceed to the generalized PDDNLS equation. Such a generalization can be written as

$$
\frac{\partial \psi}{\partial \tau}=-i v \psi-i|\psi|^{2} \psi-i \frac{\partial^{2} \psi}{\partial X^{2}}-\mu \psi+\gamma \bar{\psi}+\mathcal{N}_{\psi},
$$

with additional terms $\mathcal{N}_{\psi}$ produced by the weak-nonlinearity expansion of underlying equations of the sine-Gordon type $[61,64]$. Thus, at order $\gamma^{5 / 2}$ of the expansion, one 
can derive, in the general case,

$$
\mathcal{N}_{\psi}=\gamma\left(b \bar{\psi}|\psi|^{4}+\delta \psi^{3}+\beta \psi^{3}|\psi|^{2}\right)+i a \psi|\psi|^{4}+\eta \frac{\partial^{2} \psi}{\partial X^{2}}+c \psi|\psi|^{2}+i \zeta \bar{\psi}\left(\frac{\partial \psi}{\partial X}\right)^{2},
$$

where $\eta$ accounts for the diffusion, $c$ represents the nonlinear dissipation, $(b, \delta, \beta)$ are coefficients of the nonlinear parametric forcing, $a$ controls the quintic nonlinearity, and $\zeta$ is the coefficient of the nonlinear-drift term. The amended equation ( 8 has been employed to produce kinks, localized patterns and traveling pulses [51, 60-65, 67-76]. In fact, not all the additional terms are necessary to secure the stability of the uniform states inside of the Arnold tongue [71]. Note that, in the case of the sine-Gordon equation, the expansion at order $\gamma^{5 / 2}$ yields $\eta=0, c=0, \alpha=1 / 2, \delta=-1 / 6, a=1 / 6$, $b=1 / 12, \beta=-1 / 24$, and $\zeta=0[65]$.

We here focus on the case of $\eta=0$ and $c=0$, as effects of the terms $\sim \eta$ and $c$ have been studied before [71-73]. Besides, the effect of $\zeta$ is neglected, as it is known too, inducing traveling solutions [74]. Then, it is easy to find that the squared amplitude of the uniform-state solution, $|\psi|^{2}$, is determined by equation

$$
\gamma^{2}=\frac{\mu^{2}}{\left[1+(\delta+\alpha)|\psi|^{2}+(\beta+b)|\psi|^{4}\right]^{2}}+\frac{\left(v+|\psi|^{2}-a|\psi|^{4}\right)^{2}}{\left[1+(\alpha-\delta)|\psi|^{2}+(b-\beta)|\psi|^{4}\right]^{2}} .
$$

To study small-amplitude patterns generated by the extended PDDNLS equation, the coefficients of $\mathcal{N}_{\psi}$ in Eq. (9) are introduced as free parameters. In particular, numerical results are displayed below for $b=1 / 12, \delta=4 / 15, \beta=-1 / 24, \alpha=-0.65$, $a=1 / 6$, and $\mu=0.275$, which make it possible to produce generic findings.

\subsection{Indicators of complexity and transition between dynamical regimes}

To characterize the dynamics produced by Eq. (8), we use standard indicators, one of which is the time-dependent energy. It is often used to study non-regular dynamics in fluids, optics [75-80] and other physical systems [81-83]:

$$
Q(\tau)=\frac{1}{2 L} \int_{-L}^{+L}|\psi(\tau, X)|^{2} d X
$$

where $2 L$ is the system's size. The simple uniform stationary regime has $Q=$ const, and it is a (quasi-)periodic function of time in the case of (quasi-)periodic dynamics. An apparently random time dependence of $Q(\tau)$ corresponds to chaotic behavior.

To categorize different dynamical regimes, we also use the power spectrum of the amplitude, $S_{Q}(f)=|\mathfrak{F}(f)|^{2}$, where $\mathfrak{F}$ is the Fourier transform of $Q(\tau)$,

$$
\mathfrak{F}(f)=\frac{1}{\sqrt{2 \pi}} \int_{0}^{\tau_{\max }} Q(\tau) \exp (-i f \tau) d \tau
$$

If the time series is regular, function $S_{Q}(f)$ has a finite number of quasi-discrete peaks. On the contrary, the spectrum is essentially continuous if the series is chaotic [84]. In 


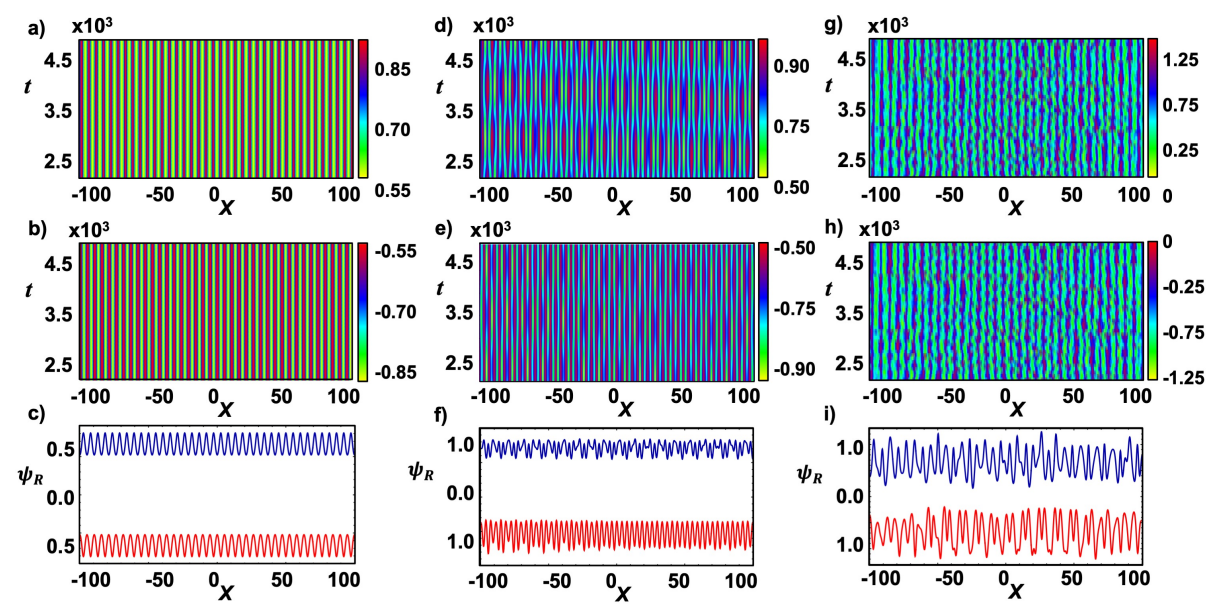

Figure 4: The spatiotemporal evolution of the real part of $\psi$ in established dynamical states shown within a time interval $\Delta \tau=2000$ (the first and second rows), and snapshots of the respective spatial patterns at a certain time (the third row). a), b) and c): Two regular small-amplitude patterns $\psi_{+/-}$(in- and out-of-phase ones, see the text) at $\gamma=0.85$ and $v=-0.25$, with $\lambda_{\max +}=-1.853 \times 10^{-3}$ and $\lambda_{\max -}=-1.852 \times 10^{-3}$. d), e) and f): Quasi-periodic states $\psi_{+/-}$at $\gamma=0.85$ and $v=-0.45$, with $\lambda_{\max +}=4.92 \times 10^{-4}$ and $\left.\left.\lambda_{\max -}=5.13 \times 10^{-4} . \mathrm{g}\right), \mathrm{h}\right)$ and i): Small-amplitude chaotic patterns $\psi_{+/-}$at $\gamma=0.85$ and $v=-0.55$, with $\lambda_{\max +}=1.288 \times 10^{-2}$ and $\lambda_{\max -}=1.359 \times 10^{-2}$. Fixed parameters are $b=1 / 12, \delta=4 / 15, \beta=-1 / 24$, $\alpha=-0.65, a=1 / 6$ and $\mu=0.275$.

addition, to characterize spatial fluctuations of field $\psi$, we also use the time average of the local density,

$$
\mathcal{L}(x)=\frac{1}{T-T_{0}} \int_{T_{0}}^{T}|\psi(\tau, X)|^{2} d \tau,
$$

and its spatial power spectrum,

$$
S_{\mathcal{L}}(k)=\int_{-L}^{+L} S_{\mathcal{L}}(k) \exp (-i k X) d X
$$

at a set of points in the frequency space $k=\left(k_{1}, \ldots, k_{n}\right)$. Similar to the temporal power spectrum, its spatial counterpart, $S_{\mathcal{L}}(k)$, helps to quantify different types of the spatial behavior of the system.

Further, a well-known potent indicator of the dynamics is the largest Lyapunov exponent $[66,85-106]$

$$
\lambda_{\max }=\lim _{\tau \rightarrow \infty}\left[\frac{1}{\tau} \ln \left(\frac{\|\delta \psi(\tau, x)\|}{\left\|\delta \psi_{0}\right\|}\right)\right],
$$

where $\|\bullet\|$ stands for the quadratic norm, and $\delta \psi(\tau, x)$ is a numerically generated solution of the linearized equation, $\partial(\delta \psi) / \partial \tau=\overline{\mathbf{J}} \cdot \delta \psi$, with $\overline{\mathbf{J}}$ being he Jacobian matrix of Eq. (8). We remark that $\lambda_{\max }$ predicts how fast distance $\delta \psi$ between two initially close trajectories of field $\psi$ grows in the course of the evolution. Namely, if the system is chaotic with $\lambda_{\max }>0$, two configurations, that were close at $\tau=\tau_{0}$, separate in phase space exponentially fast. On the other hand, in the case of $\lambda_{\max }<0$ they converge to a 

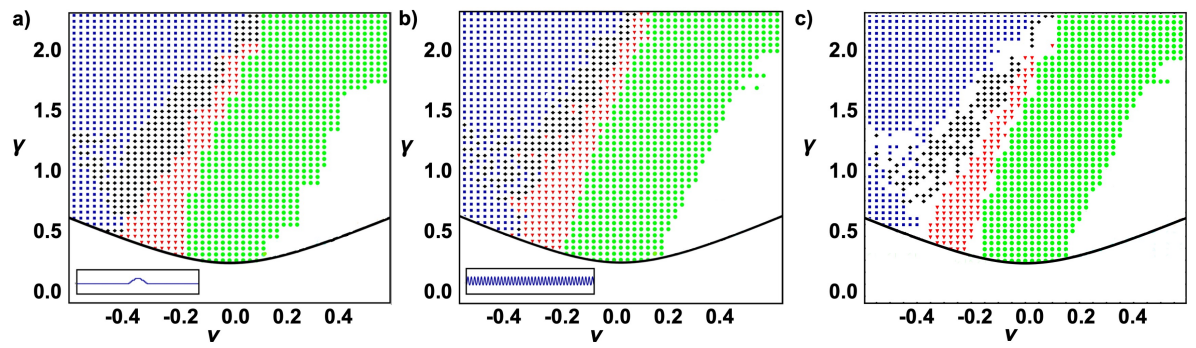

Figure 5: Phase diagrams in the $(\gamma, v)$ parameter plane for different initial conditions shown in the insets. a): A flat profile with a bump; b) a spatially-periodic profile. Green circles $(\bullet)$ correspond to the spatially uniform state, red triangles $(\boldsymbol{\nabla})$ denote stationary small-amplitude states, blue squares $(\boldsymbol{\square})$ the small-amplitude chaotic pattern, and black diamonds ( $\bullet$ correspond to quasi-periodic states with a small amplitude. c) Coincidence regions, in which the different initial conditions, used in diagrams a) and b), produce identical final states. The fixed parameters are the same as in Fig. 4.

stationary attractor. The marginal case, $\lambda_{\max }=0$, corresponds to time-periodic, quasiperiodic, or complex dynamics without exponential sensibility to the variation of initial conditions.

\section{The quasi-periodic route to spatiotemporal chaos in the generalized PDDNLS equation}

\subsection{Numerically obtained patterns}

A variable-step fifth-order Runge-Kutta scheme [107] has been used for simulations of Eq. (8) with the precision of $10^{-7}$. The spatial derivatives were approximated by means of the second-order central finite-difference method, and the Neumann's boundary conditions were used. Typical the half-size $L=100$ and $N=1500$ lattice points imply that the mesh size of the numerical scheme is $\Delta x=200 / 1500 \approx 0.13$. Sensitivity of the findings to the discretization has been verified by changing $N$ and $\Delta x$. Variation of the domain size $L$ was used to verify that finite-size effects do not affect the results. We have used two essentially different initial conditions, as shown below. The integration was performed over sufficiently long times (up to $\tau=4.8 \times 10^{3}$, to check that transients have faded out.

For the analysis of spatial patterns we present in Fig. 4 the spatiotemporal evolution of the real part of the wave field, $\psi_{\mathrm{R}} \equiv \operatorname{Re}\{\psi(x, \tau)\}$. Starting close to the tip of the Arnold tongue, which implies the consideration of a small detuning $(\gamma=0.85)$ and the forcing strength slightly greater than the dissipation $(v=-0.25$, see Eq. (6)). The regular inphase and out-of-phase states ( $\psi_{ \pm}$, with $\psi_{\mathrm{R}}>0$ and $\psi_{\mathrm{R}}<0$, respectively) are observed in Figs. 4 a)-c). In these panels the spatial periodicity observed corresponds to the real part of $\exp (i k x)$ with some $k$, while $|\psi|$ remains constant. The values of $\lambda_{\max }$ for these regular patterns are negative: $\lambda_{\max +}=-1.853 \times 10^{-3}$ and $\lambda_{\max -}=-1.852 \times 10^{-3}$, respectively.

Following the decrease of the detuning and/or the increase of the forcing strength, the pattern exhibits an oscillatory instability, which gives rise to standing waves. Figures $4 \mathrm{~d}$ )-f) show the corresponding temporarily quasi-periodic states at $\gamma=0.85$ and 

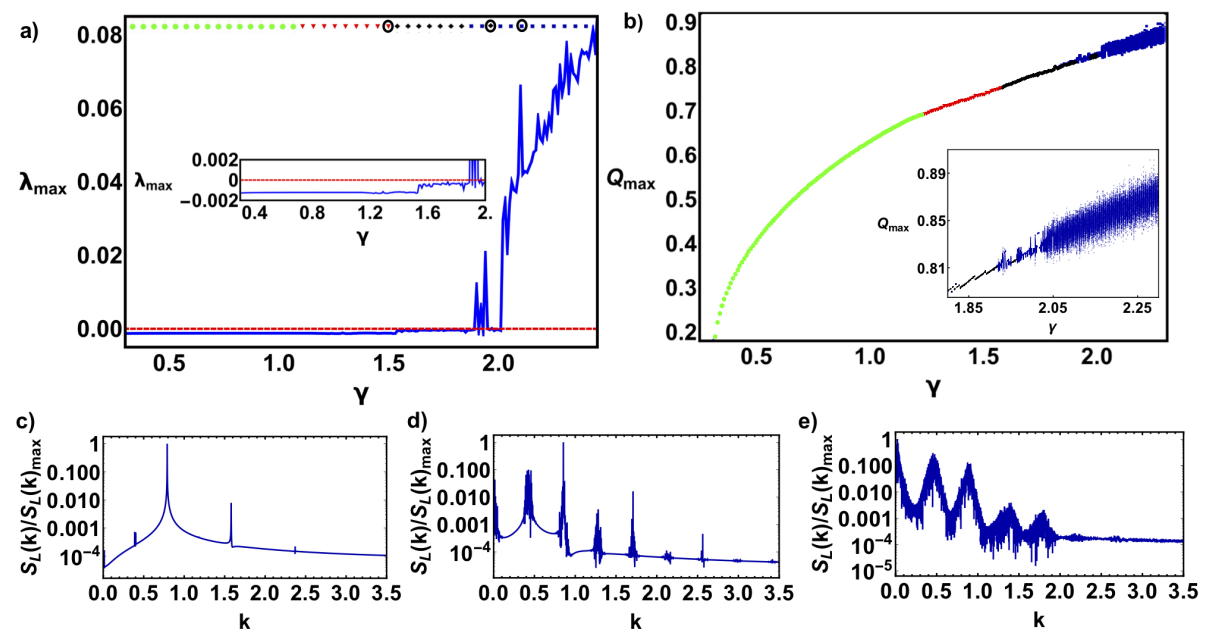

Figure 6: Characterization of the route to spatiotemporal chaos produced by simulations of Eq. (8), is shown by means of dynamical indicators. a) $\lambda_{\max }$, for $v=-0.1$, and b) $Q_{\max }$, for $v=+0.1$, as a function of the forcing strength, $\gamma$, see Eqs. (11) and (15). The insets are zoomed-in versions of the main plots. The meaning of the symbols is the same as in Fig. 5: green circles correspond to spatially uniform states, red triangles to stationary small-amplitude ones, and blue squares to the small-amplitude chaotic pattern. Panel c) shows the spatial Fourier spectrum $S_{\mathcal{L}}(k)$ (see Eq. (14)) for a regular state at $\gamma=1.50$, d) a temporarily quasi-periodic state at $\gamma=1.95$, and e) a chaotic one at $\gamma=2.1$.

$v=-0.45$, with $\lambda_{\max +}=4.92 \times 10^{-4}$ and $\lambda_{\max -}=5.13 \times 10^{-4}$ (actually, these values may be considered as a numerical zero). Changing the parameters further in the same direction, the standing wave exhibits an oscillatory instability, producing complex spatiotemporal patterns. Figures $4 \mathrm{~g}$ )-i) display typical examples of the chaotic spatiotemporal evolution of the complex patterns, accounted for by $\lambda_{\max }>0$.

Figure 5 shows phase diagrams inside the Arnold tongue for two different initial conditions (see insets): a) a flat profile with a bump, and b) a small-amplitude periodic pattern. The two respective diagrams are similar with a few differences, see the comparison between them in panel c) which displays regions in which both initial conditions produce the same final state. In the case of the small-amplitude spatially periodic initial condition, the region of the uniform state (green circles) is somewhat smaller, and the distribution of regions of temporarily stationary (red triangles), quasi-periodic (black diamonds), and chaotic (blue squares) patterns is shifted to smaller values of detuning $v$, while quasi-periodic states appear at larger values of $\gamma$. In both cases, the dynamical chaos occupies the upper left corner, while near to the zero-detuning line, $v=0$, the uniform state is the dominant one. Thus, the small-amplitude chaos is equally likely to be generated by both initial conditions.

\subsection{Dynamics inside the Arnold tongue}

Panel a) of Figure 6 presents the dependence of $\lambda_{\max }$ on the forcing strength in the case of a spatially periodic pattern chosen as initial condition. In this plot, we observe 
the transition from a stationary solution (uniform or patterned one) to an oscillatory one (the standing wave), which then turns into a spatiotemporal chaotic pattern, following the increase of the forcing strength, $\gamma$. The growth of $\lambda_{\max }$ with the subsequent increase of $\gamma$ indicates that the chaotic spatiotemporal pattern becomes more complex. Furthermore, around $\gamma \approx 2.2$, we observe an abrupt transition from $\lambda_{\max }>0$ to small islands with $\lambda_{\max }=0$, which corresponds to a phenomenon known as the crisis of a strange attractor [84].

Further, the patterns are characterized by the plot for $Q_{\max }(\gamma)$ as a function of the forcing strength $\gamma$. Figure $6 \mathrm{~b}$ ) displays it at $v=0.1$. It is produced by repeatedly picking the maximum value of the energy function $Q(\tau)$, see Eq. (11), from temporal intervals after the disappearance of transient features in the simulated evolution. The system is stationary or periodic if there is a unique value of $Q_{\max }$, while a continuous distribution of $Q_{\max }$ in a finite interval implies temporal quasi-periodicity or chaos. This figure shows that energy $Q$ strongly depends on forcing $\gamma$, showing several transitions between regular and chaotic states, denoted by the same symbols as in Fig. 5. The transitions are shown in greater detail in the inset, where the plot is blown up by a factor of 5.4. The diagrams for $\lambda_{\max }$ and $Q_{\max }$ provide mutually complimentary descriptions of the oscillatory, quasi-periodic, and chaotic behavior. Note that the plot of $Q_{\max }$ is represented by a thin curve at $\gamma<1.8$, becoming fuzzy at larger values of $\gamma$. In particular, alternation of islands, from chaos to regularity, is observed in the range of $1.92<\gamma<2.0$. The system becomes chaotic again at $\gamma>2.0$ through an abrupt transition, and remains chaotic at larger values of $\gamma$ considered here.

To grasp the route to chaos in a clearer form, we have computed the spatial Fourier spectrum $S_{L}(k)$, as per Eq. (14). Figure 6 shows $S_{L}(k)$ for the spatially uniform state at $\gamma=1.50$ in panel c), for a temporally quasi-periodic one at $\gamma=1.95 \mathrm{in} \mathrm{d}$ ), and for a chaotic state at $\gamma=2.1 \mathrm{in} \mathrm{e})$. Increase of the number of frequency peaks in the spectrum is observed as $\gamma$ increases at a fixed value of the detuning, $v=-0.1$. These plots corroborate that Eq. (8) produces a standing wave with a well-defined wavenumber and its harmonics in Fig. 6 c). As the forcing strength increases, the system generates incommensurate wavenumbers, which causes the spectral peaks to spread, see Fig. 6 d). Further increase of the strength makes the former sharp peaks broad in Fig. 6 e), which is a hallmark of spatial chaos.

Further understanding of these patterns is obtained by considering the temporal evolution of the energy function $Q(t)$, defined by Eq. (11), and the corresponding Fourier power spectrum, $S_{Q}(f)$. Panel a) of Fig. 7 shows $S_{Q}(f)$ for a quasi-periodic pattern at $\gamma=1.89$ and $v=-0.1$, with $\lambda_{\max }=-3.234 \times 10^{-4}$. The spectrum features a set of discrete peaks at particular frequencies. Accordingly, the inset shows regular evolution of $Q(t)$. On the other hand, panel b) of Fig. 7 exhibits a chaotic state at $\gamma=0.85$ and $v=-0.55$, with $\lambda_{\max }=1.288 \times 10^{-2}$ showing a continuous spectrum $S_{Q}(f)$, typical for chaotic states, with irregular evolution of $Q(t)$ in the respective inset. Thus, the transition to the spatiotemporal chaos proceeds through an intermediate stage which features the temporal quasi-periodicity. 

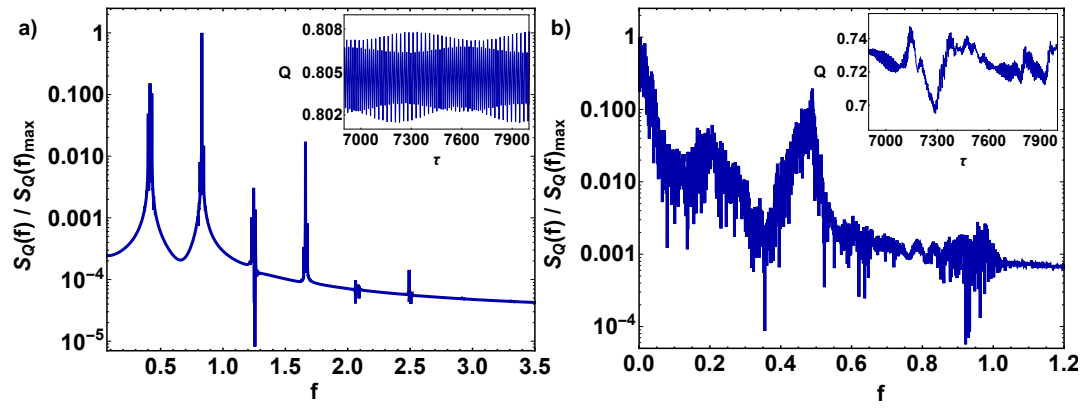

Figure 7: The power spectrum $S_{Q}(f)$ of the norm (energy) $Q(t)$, defined as per Eq. (11). a) A quasi-periodic state at $\gamma=1.89$ and $v=-0.1$. b) A chaotic state at $\gamma=0.85$ and $v=-0.55$. The insets show the respective plots of $Q(t)$.

\section{Conclusions}

In this work, we have investigated the transition to spatiotemporal chaos from small-amplitude patterns in damped driven systems close to the parametric resonance. Starting from the continuum limit of a chain of driven pendula, which is based on the equation of the sine-Gordon type, the analysis is performed in the framework of the generalized PDDNLS (parametrically driven damped nonlinear Schrödinger) equation, which is produced by the small-amplitude expansion of the underlying sine-Gordon equation. The systematic numerical analysis demonstrates that the onset of the spatiotemporal chaos proceeds through an intermediate temporally-quasi-periodic route. We use the generalized PDDNLS equation (8), which contains higher-order nonlinear terms, because the standard PDDNLS model, Eq. (5), fails to produce stable spatially uniform solutions, which play a crucial role in obtaining localized states. In particular, we have found the small-amplitude patterns that are stationary, quasi-periodic, or chaotic in time depending on the values of the strength of the parametric forcing, $\gamma$, and resonance detuning, $v$. We have concentrated on chaotic solutions, using different tools to characterize their dynamical behavior, such as the time dependence of the norm (energy) $Q$, the corresponding power spectrum, and the maximum Lyapunov exponent. Small-amplitude chaotic patterns exist inside of the Arnold tongue, competing with regular ones. Varying $\gamma$ at constant $v$ or vice versa leads in various transitions between these states. There are well confined chaotic patterns and others, in which the chaotic area penetrates the homogeneous one, following the increase of $\gamma$.

The universal nature of the present model suggests that similar scenarios of the transition to the small-amplitude spatiotemporal chaos may be expected in physical settings such as vertically oscillating fluid layers, magnetic systems, forced nonlinear lattices, and optical waveguides. A promising direction for the extension of this work is to extend it to the two-dimensional case. 


\section{Acknowledgements}

LMP and DL appreciate the hospitality of the MPI-P (Mainz) during their stay in Germany. LMP, PD and DL acknowledge partial financial support from FONDECYT 1180905. MGC acknowledges partial financial support from Millennium Institute for Research in Optics, ANID-Millennium Science Initiative Program-ICN17_012 and FONDECYT 1180903. JAV and DL acknowledge partial financial support from Centers of excellence with BASAL/CONICYT financing, Grant AFB180001, CEDENNA. The work of BAM is supported, in part, by the Israel Science Foundation through grant No. 1286/17. This author also acknowledges support from Instituto de Alta Investigación, Universidad de Tarapacá (Arica, Chile).

\section{References}

\section{References}

[1] L. D Landau and E. M. Lifshitz, Mechanics (Pergamon Press, 1976).

[2] A. Frova and M. Marenzana, Thus Spoke Galileo: The Great Scientist's Ideas and their Relevance to the Present Day, (Oxford University Press, 2006).

[3] M. Faraday, Philos. Trans. R. Soc. London 121, 299 (1831).

[4] G. Nicolis and I. Prigogine, Self-Organization in Nonequilibrium Systems, J. Wiley and Sons, New York, 1977.

[5] H. Haken, Physics Bulletin 28, 412 (1977).

[6] M. C. Cross and P. C. Hohenberg, Rev. Mod. Phys. 65, 851 (1993).

[7] L. M. Pismen, Patterns and interfaces in dissipative dynamics (Springer, Berlin, 2006).

[8] P. Manneville, Instabilities, chaos and turbulence (Imperial college Press, London, 2004).

[9] D. Walgraef, Spatio-temporal pattern formation: with examples from physics, chemistry, and materials science, (Springer Science \& Business Media, 2012).

[10] J. D. Murray, Mathematical Biology (Springer-Verlag, Berlin, 1993).

[11] G. Nicolis, Introduction to Nonlinear Science (Cambridge University Press, Cambridge, 1995).

[12] P. Coullet, L. Gil, and J. Lega, Phys. Rev. Lett. 62, 1619 (1989).

[13] P. Coullet, L. Gil, and J. Lega, Physica D 37, 91 (1989).

[14] M. G. Clerc and N. Verschueren, Phys. Rev. E 88, 052916 (2013).

[15] H. R. Brand and R. J. Deissler, Phys. Rev. Lett. 63, 2801 (1989). 
[16] H. R. Brand and R. J. Deissler, Physica A 204, 87 (1994).

[17] R. J. Deissler and H. R. Brand, Phys. Rev. Lett. 72, 478 (1994).

[18] R. J. Deissler and H. R. Brand, Phys. Rev. E 51, R852 (1995).

[19] R. J. Deissler, and H. R. Brand, Phys. Rev. Lett. 74, 4847 (1995).

[20] C. Cartes, and O. Descalzi, Physical Review A 93, 031801 (2016)

[21] O. Descalzi and H. R. Brand, Chaos 28, 075508 (2018)

[22] O Descalzi, HR Brand, Physical Review E 101, 040201 (2020)

[23] W. Decker, W. Pesch, and A. Weber, Phys. Rev. Lett. 73, 648 (1994).

[24] B. Echebarria and H. Riecke, Phys. Rev. Lett. 84, 4838 (2000).

[25] K. E. Daniels and E. Bodenschatz, Phys. Rev. Lett. 88, 034501 (2002).

[26] M. A. Miranda and J. Burguete, Phys. Rev. E 79, 046201 (2009).

[27] P. Brunet and L. Limat, Phys. Rev. E 70, 046207 (2004).

[28] Q. Ouyang and J. M. Flesselles, Nature (London) 379, 143 (1996).

[29] Z. Qu, J. N. Weiss, and A. Garfinkel, Phys. Rev. Lett. 78, 1387 (1997).

[30] S. J. Moon, M. D. Shattuck, C. Bizon, D. I. Goldman, J. B. Swift, and H. L. Swinney, Phys. Rev. E 65, 011301 (2001).

[31] S. Q. Zhou and G. Ahlers, Phys. Rev. E 74, 046212 (2006).

[32] N. Verschueren, U. Bortolozzo, M. G. Clerc, and S. Residori, Phys. Rev. Lett. 110, 104101 (2013).

[33] M.G. Clerc, G. Gonzalez-Cortes, V. Odent, and M. Wilson, Opt. Express 24, 15478 (2016).

[34] M. G. Clerc, F. Alvarez-Garrido, and G. Gonzalez-Cortes, Phys. Rev. Lett. 124, 164101 (2020).

[35] P. Coullet and J. Lega, Europhys. Lett. 7, 511 (1988).

[36] P. Coullet, L. Gil, and J. Lega, Phys. Rev. Lett. 62, 1619 (1989).

[37] P. Coullet, L. Gil, and J. Lega, Physica D 37, 91 (1989).

[38] G. Goren, J. P. Eckmann, and I. Procaccia, Phys. Rev. E 57, 4106 (1998).

[39] H. Chate, Nonlinearity 7, 185 (1994).

[40] F. Brochard, E. Gravier, and G. Bonhomme, Phys. Rev. E 73, 036403 (2006).

[41] K. He, Phys. Rev. Lett. 80, 696 (1998). 
[42] K. He and Abraham C.-L. Chian, Phys. Rev. E 69, 026207 (2004).

[43] E. L. Rempel and Abraham C.-L. Chian, Phys. Rev. Lett. 98, 014101 (2007).

[44] J. A. Sherratt and M. J. Smith, Physica D 241, 1671 (2012).

[45] Y. Kuramoto, Chemical Oscillations, Waves, and Turbulence (Springer, New York, 1984).

[46] J. W. Miles, J. Fluid Mech. 148, 451 (1984).

[47] I. V. Barashenkov, M. M. Bogdan, and V. I. Korobov, Europhys. Lett. 15, 113 (1991).

[48] B. A. Malomed, Nonlinearity and discreteness: Solitons in lattices, in: Emerging Frontiers in Nonlinear Science, pp. 81-110, ed. by P. G. Kevrekidis, J. Cuevas-Maraver, and A. Saxena (Springer Nature Switzerland AG: Cham, 2020).

[49] A. C. Scott, Am. J. Phys. 37, 52-61 (1969).

[50] P. Coullet, T. Firsch, and G. Sonnino, Phys. Rev. E 49, 2087 (1994).

[51] M. G. Clerc, S. Coulibaly, and D. Laroze, EPL 90, 38005 (2010).

[52] I. V. Barashenkov, E. V. Zemlyanaya, and T. C. van Heerden, Phys. Rev. E 83, 056609 (2011).

[53] N. V. Alexeeva, I. V. Barashenkov, and G. P. Tsironis, Phys. Rev. Lett. 84, 3053 (2000).

[54] I. V. Barashenkov, and E. V. Zemlyanaya, Phys. Rev. Lett. 83, 2568 (1999).

[55] E. V. Zemlyanaya, and N. V. Alexeeva, Theoretical and Mathematical Physics 159, 870 (2009).

[56] I. V. Barashenkov, E. V. Zemlyanaya, Phys. Rev. E 83, 056610 (2011).

[57] D. Urzagasti, D. Laroze, M. G. Clerc, S. Coulibaly, and H. Pleiner, J. Appl. Phys. 111, 07D111 (2012).

[58] V. S. Shchesnovich, and I. V. Barashenkov, Physica D 164, 83 (2002).

[59] V. I. Arnold, Geometrical methods in the theory of ordinary differential equations (Springer-Verlag, New York, 1983).

[60] M. G. Clerc, S. Coulibaly, and D. Laroze, EPL 97, 30006 (2012).

[61] M. G. Clerc, S. Coulibaly, and D. Laroze, Int. J. Bif. Chaos 19, 3525 (2009).

[62] M. G. Clerc, S. Coulibaly, and D. Laroze, Physica D 1239, 72 (2010)

[63] M. G. Clerc, S. Coulibaly, and D. Laroze, Phys. Rev. E 77, 056209 (2008). 
[64] M. G. Clerc, S. Coulibaly, and D. Laroze, Int. J. Bif. Chaos 19, 2717 (2009).

[65] D. Urzagasti, D. Laroze, and H. Pleiner, J. Special Topics 223, 141 (2014).

[66] A. Pikovsky, and A. Politi Lyapunov Exponents: A Tool to Explore Complex Dynamics. (Cambridge University Press, Cambridge, 2016)

[67] D. Urzagasti, A. Aramayo, and D. Laroze, Phys. Lett A 378, 2614 (2014).

[68] C. Elphick, E. Tirapegui, M. E. Brachet, P. Coullet, and G. Iooss, Physica D 29, 95 (1987).

[69] D. Urzagasti, D. Laroze, and H. Pleiner, Phys. Rev. E 95, 052216 (2017).

[70] E. Kenig, B. A. Malomed, M. C. Cross, and R. Lifshitz, Phys. Rev. E 80, 046202 (2009).

[71] I. V. Barashenkov, S. Cross, and B. A. Malomed, Phys. Rev. E 68, 056605 (2003).

[72] J. Burke, A. Yochelis, and E. Knobloch, SIAM J. Appl. Dyn. Sys. 7, 651 (2008).

[73] Y.-P. Ma, J. Burke, and E. Knobloch, Physica D 239, 1867 (2010).

[74] A. O. León, M. G. Clerc, and S. Coulibaly, Phys. Rev. E 91, 050901(R) (2015).

[75] Y. Edri, E. Meron, A. Yochelis, Physica D 410, 132501 (2020).

[76] Y. Edri, E. Meron, A. Yochelis, Chaos 30, 023120 (2020).

[77] N. Akhmediev, J. M. Soto-Crespo, and G. Town, Phys. Rev. E 63, 056602 (2001).

[78] A. Ankiewicz, N. Devine, N. Akhmediev, and J. M. Soto-Crespo, Phys. Rev. A 77, 033840 (2008).

[79] N. Akhmediev and J. M. Soto-Crespo, Phys. Lett. A 317, 287 (2003).

[80] N. Akhmediev and J. M. Soto-Crespo, Phys. Rev. E 70, 036613 (2004).

[81] O. Batiste, E. Knobloch, A. Alonso, and I. Mercader, J. Fluid Mech. 560, 149 (2006).

[82] J. Burke and E. Knobloch, Phys. Rev. E 73, 056211 (2006).

[83] J. Burke and E. Knobloch, Chaos 17, 037102 (2007).

[84] E. Ott, Chaos in Dynamical Systems (Cambridge University Press, Cambridge, 1993).

[85] A. Wolf, J. B. Swift, H. L. Swinney, and J. A. Vastano, Physica D 16, 285 (1985).

[86] J.C. Sprott, Chaos and Time-Series Analysis. (Oxford: Oxford University Press, Oxford, 2003) 
[87] D. Laroze, J. Bragard, O. J. Suarez, and H. Pleiner, IEEE Trans. Mag. 47, 3032 (2011).

[88] J. Bragard, H. Pleiner, O. J. Suarez, P. Vargas, J. A. C. Gallas, and D. Laroze, Phys. Rev. E 84, 037202 (2011).

[89] D. Laroze, D. Becerra-Alonso, J. A. C. Gallas, and H. Pleiner, IEEE Trans. Mag. 48, 3567 (2012).

[90] J. P. Eckmann, D. Ruelle, and S. Ciliberto, Phys. Rev. A 34, 4971 (1986).

[91] K. Geist, U. Parlitz, and W. Lauterborn, Prog. Theor. Phys. 83, 875 (1990).

[92] J. D. Scheel and M. C. Cross, Phys. Rev. E 74, 066301 (2006).

[93] A. Karimi and M. R. Paul, Phys. Rev. E 85, 046201 (2012).

[94] C. Bonatto and J. A. C. Gallas, Phys. Rev. Lett. 101, 054101 (2008).

[95] G. M. Ramirez-Avila and J. A. C. Gallas, Phys. Lett. A 375, 143 (2010).

[96] J. A. C. Gallas, Int. J. Bif. Chaos 20, 197 (2010).

[97] J. A. Vélez, J. Bragard, L. M. Pérez, A. M. Cabanas, O. J. Suarez, D. Laroze, and H. L. Mancini, Chaos 30, 093112 (2020).

[98] M. N. Mahmud, Z. Siri, J. A. Vélez, L. M. Pérez, D. Laroze, Chaos 30, 073109 (2020)

[99] D. Laroze and H. Pleiner, Commun. Nonlinear Sci. Numer. Simulat 26, 167 (2015);

[100] D. Laroze, P. G. Siddheshwar, and H. Pleiner, Commun. Nonlinear Sci. Numer. Simulat. 18, 2436 (2013).

[101] A. M. Cabanas, M .G. Clerc, D. Laroze, A. O. Leon, J. Magn. Magn. Mater. 476, 589 (2019).

[102] A. M. Cabanas, L. M. Pérez, D. Laroze, J. Magn. Magn. Mater. 460, 320 (2018).

[103] L. M. Pérez, J. Bragard, H. L. Mancini, J. A. C. Gallas, A. M. Cabanas, O. J. Suarez, D. Laroze, Netw. Heterog. Media 10, 209 (2015).

[104] E. Luis Brugnago, J. A. C. Gallas, M. W. Beims, Chaos 30, 083106 (2020).

[105] A.M. Cabanas, J. A. Vélez, L. M. Pérez, P. Dáz, M.G. Clerc, D. Laroze, B.A. Malomed, Chaos, Solitons and Fractals 146, 110880 (2021).

[106] P. G. Siddheshwar, Kanchana C., D. Laroze, Physics of Fluids 33, 044107 (2021).

[107] W. H. Press, S. A. Teukolsky, W. T. Vetterling, and B. P. Flannery, Numerical Recipes in FORTRAN (Cambridge University Press, UK, 1992). 